\title{
Development of short-term memory (STM) and acquisition
}

\author{
RICHARD H. BAUER \\ Department of Psychiatry, School of Medicine, University of California, Los Angeles, California 90024
}

\begin{abstract}
Nine- and 12-year-old subjects with high, above average, or average IQs were presented sequences of seven-digit numbers and asked to recall each sequence in serial order. Unknown to the subjects, the same sequence was repeated on the average of every fourth trial. The results showed that: (a) nonrepeated sequence (NRS) recall of older and more intelligent children was superior, but there was little change across trials, (b) repeated sequence (RS) recall of older children improved to a greater degree across trials, and (c) IQ, RS, and NRS recall were positively correlated. These results suggest that individual differences in STM can account for developmental differences in acquisition.
\end{abstract}

A number of studies indicate that STM and acquisition improve with age and intelligence (Belmont \& Butterfield, 1969; Goulet, 1968; Spitz, Goettler, \& Webreck, 1972). Since rehearsal is thought to maintain items in STM and with sufficient rehearsal information is transferred to a more permanent store (Atkinson \& Shiffrin, 1968; 1971), it has been suggested that more efficient rehearsal on the part of older and more intelligent children accounts for these results (Belmont \& Butterfield, 1969; Goulet, 1968). Although multistage learning and memory models suggest that the amount of information entering long-term storage is partly a function of the amount initially held in STM (Atkinson \& Shiffrin, 1968; 1971), little research has been devoted to exploring the relationship between development of STM and acquisition. On the basis of these models, one would expect that subjects who maintain more information in STM would show more rapid acquisition.

Adults have been tested in tasks measuring both STM and acquisition, but the relationship between the two was not reported. For example, Hebb (1961), Melton (1963), and others (Bower \& Winzenz, 1969; Cohen \& Johansson, 1967) demonstrated that recall of supraspan digit sequences improves when this sequence reoccurred several times among a series of other nonrepeated sequences (NRS). Recall of the NRS remains relatively constant across trials. In this task, NRS items are thought to be retrieved from STM, while acquisition on the repeated sequence (RS) is thought to be the result of information transferred and retrieved from a more permanent store (Cohen \& Johansson, 1967; Hebb, 1961; Melton, 1963). In the present experiment, the Hebb-Melton paradigm was used to investigate STM and acquisition of subjects differing in age and IQ.

This research was supported by Grant MH-24384-01. Data for this experiment was collected while the author was in the University of Houston Department of Psychology. The author gratefully acknowledges the cooperation of the Houston Independent School District.
In the present experiment, there was a 5-sec delay between the last item in the sequence and recall, and subjects were required to recall the digits in serial order. These requirements should remove information from immediate memory because the last digits in the sequence could not be recalled immediately, and the subject's own recall responses remove these items from immediate memory (Tulving \& Arbuckle, 1963). Therefore, NRS items are probably retrieved from STM. This delay should also allow additional time for organization and rehearsal.

\section{METHOD}

\section{Subjects}

Twenty-one 9-year-old and 21 12-year-old school children attending the Houston. Texas Unified School District served as subjects. Within the two age groups, children were separated into high $(\mathrm{Hi})$, above average (AA), and average (A) IQ groups. Table 1 presents information on IQ and sex for each of the six groups. Intelligence tests were administered 3-5 months prior to the experiment as part of routine testing in the Houston City schools, and IQ scores were obtained from the school records. The IQs were determined by the Otis-Lennon mental ability test (1967).

\section{Apparatus and Procedure}

Seven-digit numbers with digits from 1 to 9 were taken from a table of random numbers. Sequences with any digit appearing more than twice and those with more than two digits in ascending order were rejected. A total of 31 numbers was selected and ordered such that the 2nd, 5th, 9th, 14th, 20th, 26th, 31st, 35th, 38th, and 40th were the same sequence of digits. Thus, on the average, every fourth number was the same digit sequence, and a total of 40 trials was given. The RS was the same for all children. Each NRS occurred only once.

All subjects were tested between 9:00 a.m. and 4:00 p.m. in a quiet room located in the school building. The instructions and digits were presented to the subjects by means of a tape recorder. Instructions were as follows: "This is a game we play to see how well kids remember numbers. Listen carefully and you will hear some numbers. A few seconds after the numbers you will hear '.now' When you hear 'now,' tell me what the numbers were in the order you heard them. You do not have to repeat the numbers back as slowly as you hear them, but repeat them clearly so the person writing down what you say can understand you. O.K., we'll try it one time. Ready?"

A sequence was presented followed by "now," and the child reported the digits back. Additional instructions were as follows: "At first it may seem hard to remember the numbers but keep trying and 
Table 1

Mean IQ and Number of Subjects of Each Sex Separated into High, Above Average, and Average IQ Groups

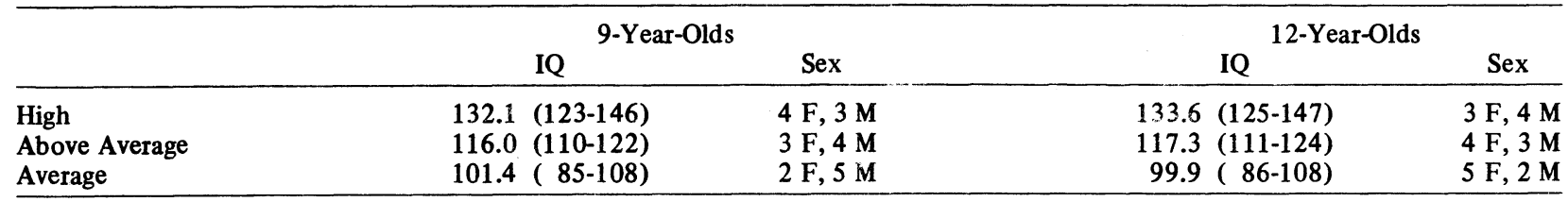

Numbers in parentheses refer to the range.

you will see it gets easier. O.K., let's try it again. Remember, when you hear 'now,' tell me the numbers in the order you heard them." Subjects were then given three or more practice trials and additional instructions depending upon their particular difficulty with the task. The experimenter sat behind and slightly to the side of the child while recording the digits reported. Subjects were not allowed to use hierarchical grouping but were required to give them back as individual digits. They were not told if they were correct or incorrect nor informed that one sequence occurred more than once.

Digits were presented at approximately 1 /second in a monotone voice. The word "now" followed the last digit of the sequence by $5.0 \pm .75 \mathrm{sec}$. The first digit of the next sequence followed the word "now" by $10.0 \pm 1.0 \mathrm{sec}$. Thus, the subjects were given $10.0 \mathrm{sec}$ to repeat the sequence back.

\section{RESULTS}

The number of digits correctly recalled in the order given was scored and converted to percentage correct. Other scoring procedures were used, but they yielded similar results. The left panels of Figure 1 show the mean percentage correct for the $10 \mathrm{RS}$ trials for each group. Percentage correct $\mathrm{RS}$ recall was analyzed by a 2(age) by 3(IQ) by 10(trials) complete factorial analysis of variance, with the last factor as a repeated measures

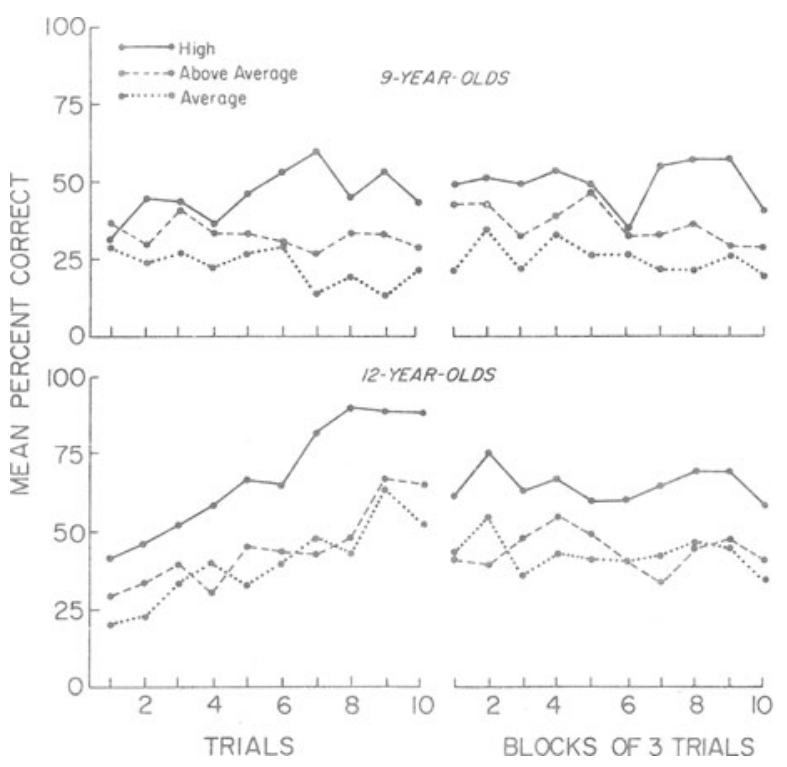

Figure 1. Mean percentage correct recall of a repeated digit sequence (left panels) and nonrepeated sequences in blocks of three trials (right panels) for high-, above-average, and average-IQ 9- and 12-year-olds. variable. This analysis indicated that recall of older children was superior to younger, $F(1,36)=21.73$, $\mathrm{p}<.001$, and that high-IQ subjects were superior to low-IQ subjects, $F(2,36)=18.31, p<.001$. The main effect for trials was also significant, $F(9,324)=10.67$, $\mathrm{p}<.001$. The Age by Trials interaction was significant, $F(9,324)=11.56, p<.001$, which indicates that across trials RS recall of older children improved to a greater degree than did recall of younger children.

Comparisons between individual treatment means by Tukey's (a) test indicated that RS recall of Hi-IQ 12-year-olds was superior to AA and A 12-year-olds ( $p<.05$ for all Tukey's tests reported), but there was no significant difference between $A A$ and $A$ 12-year-olds. All 9-year-old groups were significantly different from each other. Comparisons of 9- and 12-year-olds revealed that Hi-IQ 12-year-olds were superior to all 9-year-olds. Above average and A 12-year-olds were superior to AA and A 9-year-olds, but there was no significant difference between AA and A 12-year-olds and $\mathrm{Hi}$ 9-year-olds.

The right panels of Figure 1 present mean percentage correct NRS recall in blocks of three trials for each of the six groups. The percentage correct NRS recall was analyzed by a 2 (age) by 3 (IQ) by 10 (blocks of three trials each) complete factorial analysis of variance, with the last factor as a repeated measures variable. This analysis showed that older children were superior to younger children, $F(1,36)=11.31$, $\mathrm{p}<.001$ and that more intelligent children were superior to less intelligent, $F(2,36)=12.88, p<.001$. There was also a significant trials effect, $F(9,324)=3.01, p<.01$, but inspection of Figure 1 reveals no discernible trend. All interaction effects were not significant.

Comparisons between individual treatment means by Tukey's (a) test indicated that NRS recall of Hi-IQ 12-year-olds was superior to AA and A 12-year-olds, but there was no significant difference between AA and A 12-yeár-olds. All 9-year-old groups were significantly different from each other. Comparisons between different age groups showed that Hi-IQ 12-year-olds were superior to all 9-year-olds. High-IQ 9-year-olds were superior to A 12-year-olds but did not differ significantly from AA 12-year-olds. Above average 12-year-olds were superior to AA and A 9-year-olds.

Inspection of Figure 1 indicates that RS recall of all groups was lower on the first trial than NRS recall. 
Table 2

Correlation Matrix for Two Age Groups

\begin{tabular}{llcc}
\hline & & $\begin{array}{c}\text { Non- } \\
\text { Repeated } \\
\text { Sequence }\end{array}$ & $\begin{array}{c}\text { repeated } \\
\text { Sequence }\end{array}$ \\
\hline 9-Year-Olds & IQ & $.59 *$ & $.66^{*}$ \\
& Repeated Sequences & & $.64^{*}$ \\
12 -Year-Olds & IQ & $.64 *$ & $.56 *$ \\
& Repeated Sequences & & $.84^{*}$ \\
\hline
\end{tabular}

${ }^{*} p<.005, d f=19$

These results are probably due to the fact that, by random selection, the $\mathrm{RS}$ was more difficult than the NRS

The relationship between IQ, RS, and NRS recall was examined by Pearson product-moment correlations. These correlations are presented in Table 2. As can be seen, for both 9- and 12-year-olds there were significant positive correlations between each of the three variables.

\section{DISCUSSION}

In general, the present results indicate that older and more intelligent children recalled more NRS items, and older children showed a greater RS acquisition effect than younger children. The finding that NRS recall of older and more intelligent children was superior to that of younger and less intelligent and the positive correlations between IQ and NRS recall were expected because similar results have been reported previously (Munn, 1965; Terman \& Merrill, 1960).

Studies comparing different age and intelligence groups have supported the hypothesis that these variables influence acquisition but not forgetting rate (e.g., Belmont \& Butterfield, 1969). Therefore, differential forgetting in the delay between the last item and recall does not appear to account for the recall differences found in the present study.

The finding that older and more intelligent child ren recalled more NRS items indicates that the number of items maintained in STM increases with age and intelligence. However, NRS recall changes very little across trials, suggesting that the number of items maintained in STM remains relatively constant with practice. A number of reports indicate that older and more intelligent children are more likely to use various strategies, such as rehearsal and organization, to maintain information in STM (Belmont \& Butterfield, 1969; Spitz et al., 1972). Thus, superior NRS recall of older and more intelligent child ren is probably due to the use of more efficient learning and memory strategies on the part of older children.

The present results indicate that younger children not only recall fewer NRS items but show slower RS acquisition. The correlations between NRS and RS recall were also significant. In accord with multistage models of learning, these results suggest that the amount of information maintained in STM is an important factor for rate of acquisition. They also suggest that individual differences in STM may account for individual differences in acquisition.

Melton (1963) demonstrated that increasing the number of NRS items between the RS reduces the RS acquisition rate. Since the digit span of younger child ren is exceeded to a greater degree, the between repetition retroactive interference would be greater for younger children. Although this source of interference may be responsible for the slower RS acquisition of younger children, the finding that there was no NRS-recall decrement across trials suggests that this may not be an important factor.

\section{REFERENCES}

Atkinson, R. C., \& Shiffrin, R. M. Human memory: A proposed system and its control processes. In K. W. Spence \& J. T. Spence (Eds.), The psychology of learning and motivation (Vol. 2). New York: Academic Press, 1968.

Atrinson, R. C., \& Shiffrin, R. M. The control of short-term memory. Scientific American, 1971, 224, 82-90.

Belmont, J. M., \& Butterfield, E. C. The relations of short-term memory to development and intelligence. In L. P. Lipsitt \& H. W. Reese (Eds.), Advances in child development and behavior. New York: Academic Press, 1969.

Bower, G. H., \& Winzenz, D. Group structure, coding, and memory for digit series. Journal of Experimental Psychology Monograph, 1969, 80, 1-17.

Cohen, R. L., \& Johansson, B. S. Some relevant factors in the transfer of material from short-term to long-term memory. Quarterly Journal of Experimental Psychology, 1967, 19. 300-308.

Goulet, L. R. Verbal learning and memory research with retardates: An attempt to assess developmental trends. In N. R. Ellis (Ed.), International Review of Research in Mental Retardation, 1968, 3, 97-134.

He Bв, D. O. Distinctive features of learning in the higher animal. In J. F. Delafresnaye (Ed.), Brain mechanisms and learning. New York: Oxford University Press, 1961.

Melton. A. W. Implications of short-term memory for a general theory of memory. Journal of Verbal Learning and Verbal Behavior, 1963, 2, 1-21.

MunN, N. L. The evolution and growth of human behavior (2nd ed.). Boston: Houghton-Mifflin, 1965.

Otis. A. S., \& Lennon. R. T. Otis-Lennon mental ability test, New York: Harcourt. Brace. Jovanovich, 1967.

Spitz, H. H., Goettrler, D. R., \& Webreck, C. A. Effects of two types of redundancy on visual digit span performance of retardates and varying aged normals. Developmental Psychology, 1972, 6. 92-103.

Terman, L. M., \& Merrill, M. A. Stanford-Binet intelligence scale: Manual for the third revision. Form $L-M$. Boston: Houghton-Mifflin, 1960.

Tulving. E.. \& Arbuckle, T. Y. Sources of intratrial interference in immediate recall of paired associates. Journal of Verbal Learning and Verbal Behavior, 1963, 1. 321-334.

(Received for publication September 29. 1975.) 